\title{
NEAR-LOSSLESS COMPRESSION AND PROTECTION BY TURBO SOURCE-CHANNEL (DE-)CODING USING IRREGULAR INDEX ASSIGNMENTS
}

\author{
Laurent Schmalen and Peter Vary \\ Institute of Communication Systems and Data Processing (ind) \\ RWTH Aachen University, Germany \\ \{schmalen|vary\}eind.rwth-aachen. de
}

\begin{abstract}
In this paper, we present a novel near-lossless compression scheme for scalar-quantized source codec parameters. The scheme is comparable to a Turbo source coding approach and can inherently incorporate protection against transmission errors. We show that using the concept of EXIT charts and irregular codes, a linear programming optimization problem can be formulated and the solution of this problem leads to an irregular index assignment offering the best possible compression given the considered system model and a fixed channel quality. The performance of the compression scheme is demonstrated by a simulation example.
\end{abstract}

Index Terms - Iterative Source-Channel Decoding, Irregular Codes, Near-Lossless Source Coding, Turbo Source Coding

\section{INTRODUCTION}

With the discovery of Turbo codes, channel coding close to the Shannon limit has become possible with moderate computational complexity. In the recent years, the Turbo principle of exchanging $e x$ trinsic information between separate channel decoders has also been adapted to other receiver components. To exploit the residual redundancy in source codec parameters such as scale factors or predictor coefficients for speech, audio, and video signals in a Turbo process, iterative source-channel decoding (ISCD) has been proposed in [1], [2] as a means to further improve the quality of soft decision source decoding (SDSD) [3]. This residual redundancy occurs due to nonideal source encoding resulting from, e.g., delay or complexity constraints.

In [4] and [5], it has been shown that Turbo codes can also be used as source encoders. Conventional entropy source encoders such as Huffman codes or arithmetic codes are very sensitive to transmission errors while the Turbo source coding approach automatically incorporates error protection and can adapt on the fly to changing channel conditions by increasing or decreasing the amount of artificial redundancy introduced by the channel code.

In our contribution we introduce a novel concept for nearlossless compression of scalar-quantized source codec parameters. This concept uses a joint source-channel coding approach with ISCD at the receiver, similar to the Turbo source coding principle. The inner (channel) code of the transmitter is of rate $r \geq 1$, such that the system becomes capacity-achieving [6], [7]. If this inner (channel) code is fixed, the outer code, i.e., the (redundant) index assignment of the different parameters can be matched quite well to the inner code using the principles of irregular codes [8], allowing a simple optimization using EXIT charts [9]. The concept of irregularity has

This work was supported by the European Union under Grant FP6-2002IST-C 020023-2 FlexCode been successfully applied to the ISCD system [10] by modifying the (redundant) index assignment, i.e., the assignment of bit patterns to codebook indices, of a (scalar) quantizer to get so-called irregular index assignments (IIA). These irregular index assignments extend the concept of redundant index assignments [11]. In this contribution, we show that the optimization of the irregular index assignments can be modified such that the compression ratio is maximized, leading to an efficient, flexible compression system which can easily adapt to varying channel conditions.

\section{SYSTEM MODEL}

In Fig. 1 the baseband model of the considered ISCD system is depicted. At time instant $t$ a source encoder generates a frame $\underline{u}_{t}=\left(u_{1, t}, \ldots, u_{K_{S}, t}\right)$ of $K_{S}$ unquantized source codec parameters $u_{\kappa}$ with $\kappa \in\left\{1, \ldots, K_{S}\right\}$ denoting the position in the frame. Each value $u_{\kappa}$ is individually mapped to a quantizer reproduction level $\bar{u}_{\kappa}$, with $\bar{u}_{\kappa} \in \mathbb{U}=\left\{\bar{u}^{(1)}, \ldots, \bar{u}^{(Q)}\right\}$. The set $\mathbb{U}$ denotes the quantizer codebook with a total number of $|\mathbb{U}|=Q$ codebook entries. In this paper, we restrict $Q$ to take only values which are powers of 2, i.e., $Q=2^{M}$, with $M \in \mathbb{N} \backslash\{0\}$. A unique bit pattern $\mathbf{x}_{\kappa} \in \mathbb{X}_{\kappa}=\left\{\mathbf{x}_{\kappa}^{(1)}, \ldots, \mathbf{x}_{\kappa}^{(Q)}\right\}$ of $M_{\kappa}^{*}$ bits (i.e., $\mathbb{X}_{\kappa} \subseteq \mathbb{F}_{2}^{M_{\kappa}^{*}}$, $\mathbb{F}=\{0 ; 1\}$ ), with $M_{\kappa}^{*} \geq \log _{2} Q=M$, is assigned to each quantizer level $\bar{u}_{\kappa}$ according to the index assignment $\Gamma_{\kappa}\left(\bar{u}^{(i)}\right)=\mathbf{x}^{(i)}$, $i=1, \ldots, Q$. Note that the index assignment can differ from parameter to parameter. For notational convenience we omit the time index $t$ if the meaning of the equation is non-ambiguous.

The single bits of a bit pattern $\mathbf{x}_{\kappa}$ are indicated by $x_{\kappa}(m)$, $m \in\left\{1, \ldots, M_{\kappa}^{*}\right\}$. If $M_{\kappa}^{*}>\log _{2} Q=M$, the index assignment $\Gamma_{\kappa}$ is called redundant index assignment as it introduces redundancy: More bits than actually necessary are spent to represent a quantizer reproduction level. The index assignment can be consid-

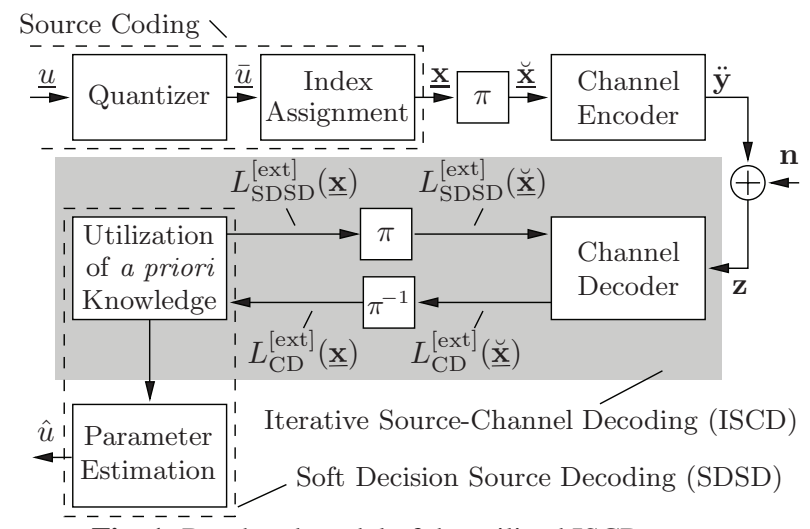

Fig. 1. Baseband model of the utilized ISCD system 
ered to be the composite function $\Gamma_{\kappa}(\bar{u})=\Gamma_{\kappa}^{\mathrm{R}}\left(\Gamma^{\mathrm{NR}}(\bar{u})\right)$. First, the function $\Gamma^{\mathrm{NR}}$ performs a non-redundant mapping of the $Q$ quantizer reproduction levels to patterns consisting of $M$ bits. Second, the function $\Gamma_{\kappa}^{\mathrm{R}}$ can be regarded as a (potentially non-linear) block code of rate $r_{\kappa}^{\mathrm{IA}}=\left(\log _{2} Q\right) / M_{\kappa}^{*}=M / M_{\kappa}^{*}$. The concept of non-linear block codes employed as redundant index assignments has been successfully utilized for the robust transmission of source parameters in, e.g., [11]. After the index assignment, $K_{S}$ bit patterns are grouped to a frame of bit patterns $\underline{\mathbf{x}}=\left(\mathbf{x}_{1}, \ldots, \mathbf{x}_{K_{S}}\right)$ consisting of $N_{X} \doteq \sum_{\kappa^{\prime}=1}^{K_{S}} M_{\kappa^{\prime}}^{*} \doteq K_{S} \cdot \bar{M}^{*}$ bits. The overall rate of the index assignment is thus

$$
\bar{r}^{\mathrm{IA}} \doteq \frac{K_{S} \cdot M}{\sum_{\kappa=1}^{K_{S}} M_{\kappa}^{*}}=\frac{M}{\bar{M}^{*}},
$$

with $\bar{M}^{*}$ the average number of bits per parameter after index assignment. The frame $\underline{x}$ of bits is re-arranged by a bit interleaver $\pi$ in a deterministic, pseudo-random like manner. The interleaved frame with $K_{S} \cdot \bar{M}^{*}$ bits is denoted as $\underline{\breve{x}}$.

For channel encoding of a frame $\breve{\mathbf{x}}$, we use a recursive convolutional code of memory $J$ and of rate $r$ CC . In general, any channel code can be used as long as the respective decoder is able to provide the required extrinsic reliabilities. For the termination of the convolutional code, $J$ tail bits are appended to $\underline{\underline{\mathrm{x}}}$. The encoded frame of length $N_{Y} \doteq \frac{1}{r \text { CC }}\left(K_{S} \cdot \bar{M}^{*}+J\right)$ is denoted by $\underline{\mathbf{y}}$. The bits $y_{k}$ of $\underline{\mathbf{y}}$ are indexed by $k \in\left\{1, \ldots, N_{Y}\right\}$. According to [6], a necessary condition for a serially concatenated system to be capacity achieving is an inner component with code rate $r^{\text {inner }} \geq 1$. This means that the channel code should be of rate $r^{\mathrm{CC}} \geq 1$, which can be achieved, e.g., by puncturing the output of the channel encoder.

Prior to transmission over the channel, the encoded bits $y_{k}$ are mapped to bipolar values $\ddot{y}_{k}$ forming a sequence $\ddot{\mathbf{y}} \in\{ \pm 1\}^{N_{Y}}$. On the channel, the modulation symbols $\ddot{y}_{k}$ (with symbol energy $E_{\mathrm{s}}=1$ ) are subject to additive white Gaussian noise (AWGN) with known power spectral density $\sigma_{n}^{2}=N_{0} / 2$.

The received symbols $z_{k}$ are transformed to $L$-values [12] prior to being evaluated in a Turbo process which exchanges extrinsic reliabilities between channel decoder (CD) and soft decision source decoder (SDSD). The channel decoder used in this paper is based on the MAP algorithm [13]. For the equations of the SDSD, we refer to the literature, e.g., [14].

\section{NEAR-LOSSLESS SOURCE CODING USING IRREGULAR INDEX ASSIGNMENTS}

It has been shown in, e.g., [4] and [5], that Turbo codes can be efficiently used as source codes, realizing a near-lossless compression scheme. Near-lossless means that the perfect reconstruction is not guaranteed. Classical entropy coding compression schemes like Huffman or arithmetic codes are able to achieve high compression ratios with moderate computational complexity, however, in the presence of channel noise, severe error propagation and synchronization losses are possible. Soft decision source decoding and iterative source-channel decoding can also be applied to entropy codes [15], with increased computational complexity however. It has been shown in [7] that with lower computational complexity at the receiver, a system utilizing fixed-length codes can achieve comparable results (in terms of reconstruction quality and symbol error rate) to a system with variable-length codes, in the case that channel noise is present.

The ISCD system using redundant index assignments introduced in Section 2 is used in this paper for realizing a near-lossless source coding system. In this case, the convolutional code is a rate $r^{\mathrm{CC}}>1$ code obtained by puncturing a rate $<1$ code and the index assign- ment has to be optimized such that a minimum number of transmitted bits $N_{Y}=\left(N_{X}+J\right) \cdot \frac{1}{r \text { CC }}$ results.

Thus, the task of the source coder is to find an index assignment which minimizes the number of transmitted bits and allows near-lossless decoding of the parameters at the receiver/decoder. The approach presented here is based on the concept of irregular index assignments [10] which extends the concept of irregular codes to the source coder. Irregular codes, originally proposed for convolutional codes [8], use several component codes of different rates in one block to obtain an outer code with overall rate $r^{\text {outer }}$. With this concept, capacity achieving codes can be easily found. The principle of irregular codes is based on the fact that the EXIT characteristic of the resulting code corresponds to the weighted sum of the component codes' characteristics. An optimization algorithm that searches for optimum weights in order to get an (almost) perfectly matching characteristic can be formulated [8].

Irregular index assignments (IIA), introduced in [10], are an extension of the irregular codes' concept. As stated in Section 2, the index assignment for the parameter $u_{\kappa}$ comprises a block code $\Gamma_{\kappa}^{\mathrm{R}}$ of rate $r_{\kappa}^{\mathrm{IA}}=\left(\log _{2} Q\right) / M_{\kappa}^{*}=M / M_{\kappa}^{*}$. Instead of using the same amount of bit redundancy $M_{\kappa}^{*}=\bar{M}^{*}$ for each parameter in order to achieve an overall rate $M / \bar{M}^{*}$ outer encoding, we use the concept of irregular codes and vary $M_{\kappa}^{*}$ for each parameter while keeping $\bar{M}^{*}$ constant. This allows us to optimize the index assignments and to get an SDSD EXIT characteristic which matches the channel decoder characteristic considerably well.

In order to perform source coding, the optimization goal is a different one as in [8] and [10]. The index assignments which have a high rate (and thus a small number of output bits) shall be preferred. Therefore, the goal of the optimization is to find an EXIT characteristic which results in the smallest number of transmitted bits with the constraint that an open decoding tunnel exists. Therefore, the weighting factors $\mathbf{w}=\left(w_{1}, \ldots, w_{L}\right)^{T}$ of the $L$ different utilized index assignments need to be chosen such that the weights $w_{\ell}$ corresponding to high-rate index assignments are preferred. The optimization goal is in fact to minimize the number of bits $N_{X}=K_{S} \bar{M}^{*}$ after index assignment. The optimized weights $\mathbf{w}$ indicate the proportions of bits after index assignment and from these, the different $K_{S, \ell}$, i.e., the number of parameters encoded with the $\ell$ 'th index assignment, can be determined. The number of resulting output bits after encoding a portion of $K_{S, \ell}$ parameters with an index assignment of rate $r_{\ell}^{\mathrm{IA}}$ amounts to

$$
K_{S, \ell} \frac{M}{r_{\ell}^{\mathrm{IA}}}=w_{\ell} N_{X}
$$

and it holds $\sum_{\ell} K_{S, \ell} \stackrel{!}{=} K_{S}$. Rewriting (2) to

$$
N_{X} w_{\ell} r_{\ell}^{\mathrm{IA}}=M K_{S, \ell}
$$

and summing up over all $L$ different index assignments leads to

$$
N_{X} \sum_{\ell=1}^{L} r_{\ell}^{\mathrm{IA}} w_{\ell}=M \sum_{\ell=1}^{L} K_{S, \ell}
$$

which can be rewritten as

$$
N_{X} \mathbf{r}^{T} \mathbf{w}=M K_{S} \Rightarrow N_{X}=\frac{M K_{S}}{\mathbf{r}^{T} \mathbf{w}},
$$

with $\mathbf{r}=\left(r_{1}^{\mathrm{IA}}, \ldots, r_{L}^{\mathrm{IA}}\right)^{T}$. As $K_{S}$ and $M$ are constant, minimizing the number of total bits $N_{X}$ corresponds to maximizing $\mathbf{r}^{T} \mathbf{w}$. This means that the task of optimizing the irregular index assignment such that decoding is still possible and resulting with a minimum number of output bits $N_{X}$ can be formulated as a linear programming optimization problem with

$$
\mathbf{w}_{\text {opt }}=\arg \max _{\mathbf{w}} \mathbf{r}^{T} \mathbf{w}=\arg \min _{\mathbf{w}}\left(-\mathbf{r}^{T} \mathbf{w}\right)
$$


subject to

$$
\begin{aligned}
\mathbf{C} \cdot \mathbf{w} & >\mathbf{d}+\mathbf{t}^{\prime} \\
0 \leq w_{\ell} & \leq 1 \quad \forall \ell \in\{1, \ldots, L\} \\
\sum_{\ell=1}^{L} w_{\ell} & =1
\end{aligned}
$$

with $\mathbf{C} \doteq\left(\mathbf{c}_{1}, \ldots, \mathbf{c}_{L}\right)$ composed of $\mathbf{c}_{\ell} \doteq\left(c_{\ell, 1}, \ldots, c_{\ell, P}\right)^{T}$, which consists of $P$ sample points of the characteristic $\mathcal{C}_{\mathrm{SDSD}, \ell}$ of a specific index assignment $\Gamma_{\ell}^{\mathrm{R}}$. The vector $\mathbf{d}$ consists of $P$ sample points of the inverse channel code EXIT characteristic $\mathcal{C}_{\mathrm{CD}}^{-1}$, measured at the channel quality for which the system is optimized. The constraint (7) ensures that an open decoding tunnel is present. In (7), the vector $\mathbf{t}^{\prime}$ denotes an offset vector which is chosen such that a larger open decoding tunnel is present, leading to better convergence properties at the receiver. In fact, the constraint $\mathbf{C w}>\mathbf{d}$ would only guarantee an infinitely small decoding tunnel which could only be exploited with an infinite block size $\left(K_{S} \rightarrow \infty\right)$. The constraints (8) and (9) ensure that all the weighting factors $w_{\ell}$ are in the range $0 \leq w_{\ell} \leq 1$ and that $\sum_{\ell} w_{\ell}=1$. The solution to this linear programming optimization problem can be easily found using numerical methods (see, e.g., [16]).

Using the factors $\mathbf{w}_{\mathrm{opt}}$ resulting from the optimization (6), the number of parameters $K_{S, \ell}$ which are encoded with the index assignment of rate $r_{\ell}^{\mathrm{IA}}$ can be determined. An equation system with $L+1$ unknowns, consisting of the $L$ different equations (2) $(\ell=$ $1 \ldots, L)$ and $\sum_{\ell} K_{S, \ell} \stackrel{!}{=} K_{S}$ can be formulated

$$
\underbrace{\left(\begin{array}{ccccc}
w_{1} & -\frac{M}{r_{1}^{\mathrm{IA}}} & 0 & \cdots & 0 \\
w_{2} & 0 & -\frac{M}{r_{2}^{\mathrm{IA}}} & \ddots & \vdots \\
\vdots & \vdots & \ddots & \ddots & 0 \\
w_{L} & 0 & \cdots & 0 & -\frac{M}{r_{L}^{\mathrm{IA}}} \\
0 & 1 & \cdots & \cdots & 1
\end{array}\right)}_{\doteq \mathbf{F}} \cdot\left(\begin{array}{c}
N_{X} \\
K_{S, 1} \\
\vdots \\
K_{S, L}
\end{array}\right)=\left(\begin{array}{c}
0 \\
\vdots \\
0 \\
K_{S}
\end{array}\right) .
$$

The solution to (10) is given by

$$
\left(\begin{array}{c}
N_{X} \\
\mathbf{K}
\end{array}\right)=\mathbf{F}^{-1} \cdot\left(\begin{array}{c}
\mathbf{0}_{L} \\
K_{S}
\end{array}\right)
$$

with $\mathbf{K}=\left(K_{S, 1}, \ldots, K_{S, L}\right)^{T}$ and $\mathbf{0}_{L}$ denoting the length $L$ all-zeros column vector. The fractions $K_{S, \ell}$ as well $N_{X}$ can be determined by (numerically) solving (11). Note that due to its special structure, $\mathbf{F}$ has full rank and can be inverted. The value $N_{X}=K_{S} \bar{M}^{*}$ can also be determined using (5).

\section{SIMULATION EXAMPLE}

We show the concept of near-lossless source coding using irregular index assignments by a simulation example. A block consisting of $K_{S}=10000$ parameters resulting from a Gaussian distributed autoregressive source of first order (Gauss-Markov source) shall be compressed. The parameters show intra-frame correlation (i.e., the parameters in one frame are correlated) with correlation coefficient $\rho=0.9$. The parameters are quantized using a $Q=16$ level LloydMax codebook $\mathbb{U}$. The index assignments $\mathrm{BC}_{5}^{16}, \mathrm{BC}_{6}^{16}, \ldots, \mathrm{BC}_{15}^{16}$ (with $\mathrm{BC}_{M^{*}}^{Q}$ ) are generated using the design guidelines and the generator matrix given in [10].

For demonstrating the concept, we utilize a rate $r_{\text {orig }}^{\mathrm{CC}}=\frac{1}{2}$ recursive systematic convolutional code of memory $J=3$ with generator

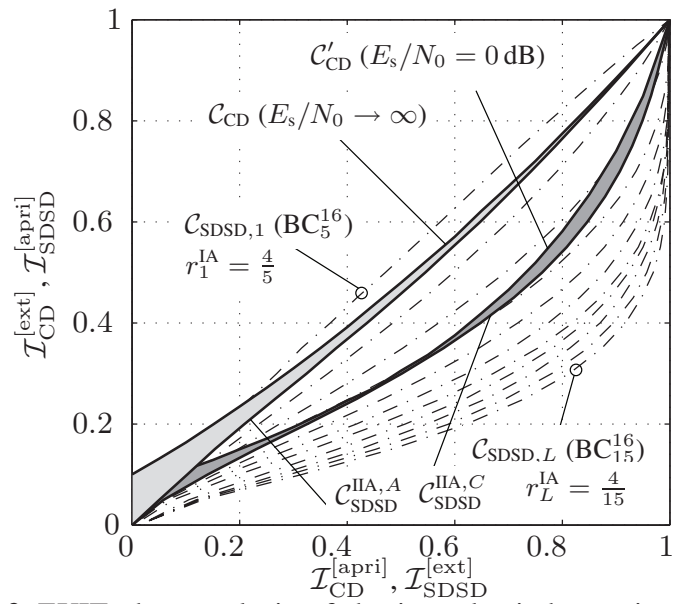

Fig. 2. EXIT chart analysis of the irregular index assignments for source coding

polynomials $G_{\text {orig }}^{\mathrm{CC}}(D)=\left(1, \frac{1}{1+D+D^{2}+D^{3}}\right)$ which is punctured to an overall rate $r^{\mathrm{CC}}=2$ code by using the puncturing matrix

$$
\mathbf{P}=\left(\begin{array}{llllllllll}
1 & 0 & 0 & 0 & 0 & 0 & 0 & 0 & 0 & 0 \\
0 & 0 & 1 & 0 & 1 & 0 & 1 & 0 & 1 & 0
\end{array}\right)
$$

The EXIT characteristic $\mathcal{C}_{\mathrm{CD}}$ of the rate $r^{\mathrm{CC}}=2$ convolutional code for perfect channel conditions (i.e., $E_{\mathrm{s}} / N_{0} \rightarrow \infty$ ) as well as the SDSD EXIT charts of the different index assignments of rates $4 / 15 \rightarrow 4 / 5$ are depicted in Fig. 2. The irregular characteristic $\mathcal{C}_{\mathrm{SDSD}}^{\mathrm{IIA}, A}$ resulting from the optimization (6) is also depicted in Fig. 2. A quite narrow open decoding tunnel is present which means that quite a large number of iterations have to be performed at the receiver. The numerical result of the optimization is given in Table 1 (Setup $A$ ): only the rate $4 / 5$ and $4 / 6$ index assignments are utilized. For $K_{S}=10000$, this leads to a total number of $N_{X}=55285$ bits after index assignment, i.e., a total number of 27644 bits after convolutional coding $(\approx 2.76$ bit per parameter). The upper limit of the reachable compression is given by the conditional entropy $H\left(\bar{U}_{\kappa} \mid \bar{U}_{\kappa-1}\right)$ which in this setup amounts to $H\left(\bar{U}_{\kappa} \mid \bar{U}_{\kappa-1}\right)=2.62$ bit per parameter leading to a minimal number of 26202 bits per block. The entropy can only be achieved if the decoding tunnel becomes infinitely narrow, however, this is not possible due to the channel decoder characteristic $\left(\mathcal{I}_{\mathrm{CD}}^{[\mathrm{ext}]}\left(\mathcal{I}_{\mathrm{CD}}^{[\mathrm{apri}]}=0\right) \approx 0.1\right)$. The utilization of a different channel code with a smaller value of $\mathcal{I}_{\mathrm{CD}}^{[\mathrm{ext}]}\left(\mathcal{I}_{\mathrm{CD}}^{[\mathrm{apri}]}=0\right)$ might lead to better compression properties.

However, the decoding tunnel might be too narrow for the decoding because of the finite block size. By selecting an offset vector $\mathbf{t}^{\prime}>\mathbf{0}_{L}$ a broader decoding tunnel can be found. The results of the optimization for this case are also given in Table 1 (Setup $B$ ).

The main advantage of the proposed system is the high flexibility. If the system shall be designed such that channel induced errors may occur, the parameter settings for the irregular index assignment can be easily adapted. As an example, we assume that the channel quality can drop down to $E_{s} / N_{0}=0 \mathrm{~dB}$. In this case, the EXIT characteristic of the channel decoder changes and an intersection occurs. The EXIT characteristic of the rate $r^{\mathrm{CC}}=2$ convolutional code for $E_{\mathrm{s}} / N_{0}=0 \mathrm{~dB}$ is also depicted in Fig. 2 and denoted by $\mathcal{C}_{\mathrm{CD}}^{\prime}$. Using this characteristic, the linear programming optimization (6) can be carried out and a new optimized EXIT curve $\mathcal{C}_{\text {SDSD }}^{\text {IIA, } C}$ results. Of course, as the channel quality becomes worse, additional artificial 
- Setup $A\left(E_{\mathrm{s}} / N_{0} \rightarrow \infty, \mathbf{t}^{\prime}=\mathbf{0}_{L}\right)$

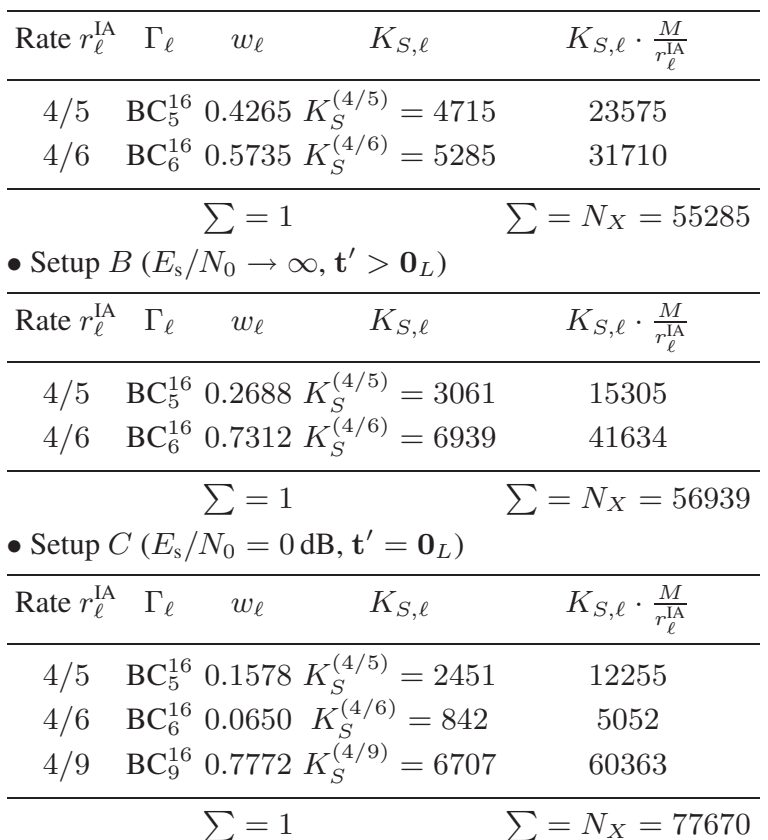

Table 1. Results of the irregular index assignment for near-lossless source coding

redundancy has to be introduced resulting in a larger block size $N_{X}$ after index assignment. The result of the optimization is also given in Table 1 (Setup $C$ ). This irregular index assignment results in a total number of $N_{X}=77670$ bits, i.e., 38837 bits after channel coding.

Figure 3 depicts the parameter SNR after reconstruction as a function of the performed iterations. For all three setups, a perfect channel quality $\left(E_{\mathrm{s}} / N_{0} \rightarrow \infty\right)$ has been studied in the example. Additionally, for Setup $C$, a channel quality of $E_{\mathrm{s}} / N_{0}=0 \mathrm{~dB}$ has been utilized. The more bits are utilized (and thus the larger the decoding tunnel is) the faster the convergence to the maximum parameter SNR of $\approx 20 \mathrm{~dB}$ (due to the quantization with codebook $\mathbb{U}$ ). For Setup $A$, it can be observed that even with 90 iterations we can only approach the maximum attainable parameter SNR. This is due to the very narrow decoding tunnel caused by $\mathbf{t}^{\prime}=\mathbf{0}_{L}$ and the finite block size of $K_{S}=10000$ parameters. In Setup $B$, with a slightly wider decoding tunnel $\left(\mathbf{t}^{\prime}>\mathbf{0}_{L}\right.$ with $\left.\max _{\ell} t_{\ell}^{\prime}=0.01\right)$ a faster convergence can be observed and the maximum parameter SNR is reached. Setup

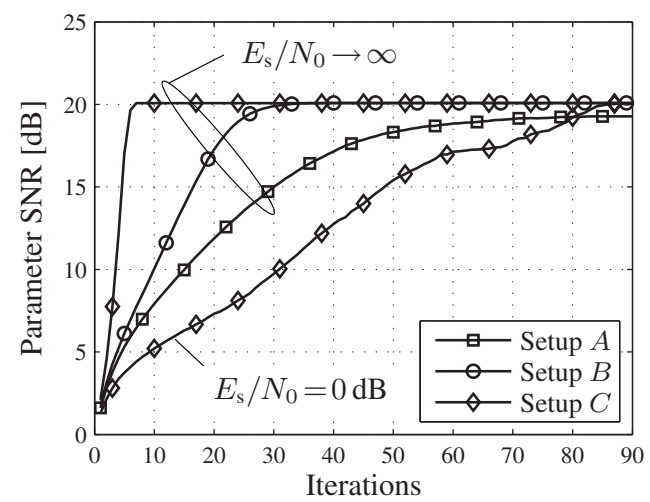

Fig. 3. Reconstruction parameter SNR for the ISCD system employing irregular index assignments
$C$ with $E_{\mathrm{s}} / N_{0}=0 \mathrm{~dB}$ shows a slower convergence than Setup $A$ because of the very narrow decoding tunnel visible in Fig. 2 (especially during the first iterations). For Setup $C$ with $E_{\mathrm{s}} / N_{0} \rightarrow \infty$ a large decoding tunnel is open. This leads to a very fast convergence (7 iterations) towards the maximum parameter SNR.

\section{CONCLUSIONS}

In this contribution we have shown that a joint source-channel coding approach with irregular index assignments and iterative decoding can be effectively utilized for near-lossless Turbo source coding. In a source coding setup, the number of transmitted bits shall be minimized. Minimizing the number of bits leads to a linear programming optimization problem which can be solved, resulting in irregular index assignments for Turbo source coding. The advantage of the proposed scheme is that it can easily cope with channel noise and can be flexibly adapted to varying channel conditions by a selection of different appropriate index assignments. The capabilities and the flexibility of the proposed source coding scheme have been demonstrated in a simulation example.

\section{REFERENCES}

[1] M. Adrat, P. Vary, and J. Spittka, "Iterative Source-Channel Decoder Using Extrinsic Information from Softbit-Source Decoding," in Proc. of IEEE ICASSP, Salt Lake City, UT, USA, May 2001.

[2] N. Görtz, "On the Iterative Approximation of Optimal Joint SourceChannel Decoding," IEEE J. Select. Areas Commun., vol. 19, no. 9, pp. 1662-1670, Sept. 2001.

[3] T. Fingscheidt and P. Vary, "Softbit Speech Decoding: A New Approach to Error Concealment," IEEE Trans. Speech Audio Processing, vol. 9, no. 3, pp. 240-251, Mar. 2001.

[4] P. Mitran and J. Bajcsy, "Turbo Source Coding: A Noise-Robust Approach to Data Compression," in Proc. of Data Compression Conference (DCC), Snowbird, UT, USA, Apr. 2002, p. 465.

[5] J. Garcia-Frias and Y. Zhao, "Compression of Binary Memoryless Sources Using Punctured Turbo Codes," IEEE Commun. Lett., vol. 6, no. 9, pp. 394-396, Sept. 2002.

[6] A. Ashikhmin, G. Kramer, and S. ten Brink, "Extrinsic Information Transfer Functions: Model and Erasure Channel Properties," IEEE Trans. Inform. Theory, vol. 50, no. 11, pp. 2657-2673, Nov. 2004.

[7] R. Thobaben, "A New Transmitter Concept for Iteratively-Decoded Source-Channel Coding Schemes," in Proc. of IEEE SPAWC, Helsinki, Finland, June 2007.

[8] M. Tüchler and J. Hagenauer, "EXIT Charts of Irregular Codes," in Proc. of Conf. on Inform. Sciences and Systems (CISS), Princeton, NJ, USA, Mar. 2002.

[9] S. ten Brink, "Convergence Behaviour of Iteratively Decoded Parallel Concatenated Codes," IEEE Trans. Commun., vol. 49, no. 10, pp. 1727-1737, Oct. 2001.

[10] L. Schmalen, P. Vary, T. Clevorn, and B. Schotsch, "Efficient Iterative Source-Channel Decoding Using Irregular Index Assignments," in Proc. of Intl. ITG Conf. on Source and Channel Coding (SCC), Ulm, Germany, Jan. 2008.

[11] T. Clevorn, P. Vary, and M. Adrat, "Iterative Source-Channel Decoding using Short Block Codes," in Proc. of IEEE ICASSP, Toulouse, France, May 2006.

[12] J. Hagenauer, E. Offer, and L. Papke, "Iterative Decoding of Binary Block and Convolutional Codes," IEEE Trans. Inform. Theory, vol. 42, no. 2, pp. 429-445, Mar. 1996.

[13] L. R. Bahl, J. Cocke, F. Jelinek, and J. Raviv, "Optimal Decoding of Linear Codes for Minimizing Symbol Error Rate," IEEE Trans. Inform. Theory, vol. 20, no. 2, pp. 284-287, Mar. 1974.

[14] M. Adrat, T. Clevorn, and L. Schmalen, "Iterative Source-Channel Decoding \& Turbo DeCodulation," in Advances in Digital Speech Transmission, R. Martin, U. Heute, and C. Antweiler, Eds., chapter 13, pp. 365-398. John Wiley \& Sons, Ltd., Jan. 2008.

[15] A. Guyader, E. Fabre, C. Guillemot, and M. Robert, "Joint SourceChannel Turbo Decoding of Entropy-Coded Sources," IEEE J. Select. Areas Commun., vol. 19, no. 9, pp. 1680-1696, Sept. 2001.

[16] P. E. Gill, W. Murray, and M. H. Wright, Numerical Linear Algebra and Optimization, vol. 1, Addison Wesley, Redwood City, 1991. 